\title{
La justificación del derecho penal en Jakobs y sus consecuencias en el ejercicio de la potestad jurisdiccional en un Estado constitucional
}

Yván Montoya Vivanco*

El presente artículo no pretende hacer una exposición de las funciones que legitiman la intervención punitiva del Estado ${ }^{1}$ sino que se limita a observar las peligrosas consecuencias que para el trabajo jurisdiccional conlleva la concepción jakobsiana sobre la función del derecho penal. La reflexión sobre este tema resulta importante no solo por la presencia cada vez más numerosa de la obra traducida del prestigioso autor alemán en el Perú, sino por la extensión progresiva de sus seguidores en nuestro país.

Existen diversas formulaciones de la teoría de la prevención general positiva las cuales podrían agruparse en dos grandes perspectivas: ${ }^{2}$ las formulaciones cuya pretensión es la legitimación del derecho penal y las que pretenden solo constituirse en un efecto que limita los excesos que produciría una finalidad meramente intimidatoria del derecho penal. Son a las primeras a las que dedicaré estas sumarias observaciones.

Günter Jakobs es probablemente el autor más representativo en la formulación de la prevención general positiva en tanto teoría legitimadora de la intervención

\footnotetext{
Abogado, egresado de la Facultad de Derecho y máster en derecho penal de la Pontificia Universidad Católica del Perú, es doctor en Derecho por la Universidad de Salamanca, España.

1 Debe recordarse que en la actualidad el derecho penal especialmente europeo continental no justifica la intervención penal del Estado sobre la base de criterios retribucionistas, sino de criterios utilitaristas referidos a la utilidad social de la pena. En ese sentido, el debate moderno entre los penalistas se centra en resaltar mejor alguna de las funciones preventivas que se le atribuyen al derecho penal: general negativa (intimidación), general positiva (fidelidad a la norma jurídica) y especial positiva (resocialización) son las predominantes en el debate del moderno derecho penal.

2 Mir Puig, Santiago. «Función fundamentadora y función limitadora de la prevención general positiva». En Poder y control. Barcelona: 1986.
} 
penal. Su planteamiento, sin embargo, no es uniforme. ${ }^{3}$ Este ha sido objeto de varias modificaciones cualitativas, pasando de una concepción de la pena centrada en sus efectos psicologistas — fidelidad ciudadana al Derecho-,${ }^{4}$ hasta su última construcción, más compleja y articulada, que asigna al derecho penal la función de garantía de las expectativas sociales esenciales frente a las conductas que cuestionan la vigencia de la norma penal, en otras palabras, la finalidad de confirmar la confianza en la vigencia de las normas pese a su ocasional infracción. ${ }^{5}$

La prevención general positiva o integradora señala, en su primera versión, que la finalidad del derecho penal no debe ser principalmente la intimidación general a los ciudadanos como potenciales delincuentes, sino «[...] reforzar la conciencia jurídica de la comunidad y su disposición por cumplir las normas». ${ }^{6}$

Son diversas y duras las críticas que ha recibido esta tesis, ${ }^{7}$ las mismas que, a su vez, el propio Jakobs ha intentado responder ${ }^{8}$ no siempre en forma convincente. No consideramos fructífero en la perspectiva de nuestro trabajo exponer todas y cada una de dichas críticas, así como todas y cada una de las réplicas a las mismas. Nuestra pretensión es solo evidenciar los aspectos que no nos resultan convincentes desde una perspectiva peruana o latinoamericana o que nos parecen todavía ambiguos de la posición de Jakobs. Solo así estaremos en mejores condiciones para exponer nuestra postura sobre las consecuencias político criminales de la (última) tesis de Jakobs sobre el ejercicio de la potestad jurisdiccional en el contexto de un Estado en el que la Constitución tiene carácter normativo o fuerza vinculante. ${ }^{?}$

\footnotetext{
Sobre las posiciones contradictorias de Jakobs ver Portilla Contrera, Guillermo. «Los excesos del formalismo jurídico neofuncionalista en el normativismo del derecho penal». En Mutaciones de Leviatán. Legitimación de los nuevos modelos penales. Madrid: Ediciones Akal, 2005, p. 58 y ss.

4 JakoBS, Günter. «Culpabilidad y prevención». En Estudios de derecho penal. Traducción de Carlos Suárez Gonzales del texto en alemán «Schuld und Prävention», Tübingen, 1976. Madrid: UAM-Civitas, 1997, p. 79.

5 JakовS, Günter. Derecho penal. Parte general. Fundamentos y teoría de la imputación. Madrid: Marcial Pons, 1995, p. 14. Hace referencia a este cambio Peñaranda Ramos, Enrique; SuÁrez Gonzáles, Carlos; Cancio Melia, Manuel. «Consideraciones sobre la teoría de la imputación de Günter Jakobs». Introducción al libro de JAKOBS, Günter. Estudios de derecho penal. Madrid: Civitas, 1997, p. 18

6 Silva Sánchez, Jesús María. Aproximaciones al Derecho Penal Contemporáneo, Bosch, Barcelona, 1992.

7 MIR PuIG, Santiago, El derecho penal en el Estado democrático de Derecho, Ariel, Barcelona, 1994.

8 Peñaranda Ramos, Enrique y Suárez Gonzales, Carlos; y Cancio Melaa, Manuel. \«Consideraciones sobre la teoría de la imputación de Günter Jakobs \». Introducción a Günter Jakobs, Estudios de Derecho Penal, Civitas, Madrid, 1997.

9 PrIETO SANCHIS, Luis. Justicia constitucional y derechos fundamentales. Madrid: Editorial Trotta, 2003, p. 116.
} 


\section{La separación entre la dogmática penal y la política criminal en Jakobs}

Creo que el primer aspecto que debe plantearse para distanciarse de las tesis preventivo integradoras (o estabilizadoras), en tanto tesis legitimadoras, es responder a las preguntas ¿qué pretendemos que pueda y deba hacer el operador jurisdiccional con el derecho penal? ¿Este solo debe manipularlo dentro del sistema positivo legal o debe trascender de él sobre la base de un control de legitimidad en función de normas iusfundamentales (Constitución)?

En otras palabras, ¿asignamos a los operadores judiciales un poder de recreación normativa - que supone aplicar una norma penal y una pena- en función de criterios político criminales con contenido valorativo o amputamos tal potestad para reservarla exclusivamente a los centros de decisión política? ¿Debe o no un juez democrático contener por vía de interpretación teleológico funcional (valorativa) los excesos de una política criminal práctica que desdibuja o se desvía del modelo de convivencia social democrática que orienta la Constitución Política de un país democrático? Nosotros optamos por una respuesta afirmativa. ${ }^{10}$ La experiencia peruana nos enseña la necesidad de contar con operadores judiciales que efectivamente se constituyan en un poder del Estado que cumpla su función de límite a los otros dos poderes constituidos: el Poder Legislativo y el Poder Judicial. Esto solo es posible con un acusado ejercicio de control de la legitimidad constitucional de las normas jurídico penales, y ello es ejercicio político criminal del derecho penal. ${ }^{11}$

Sobre estas cuestiones, la postura del profesor alemán no me resulta lo suficientemente clara. En él pueden encontrarse conviviendo dos perspectivas diferentes según las cuales sus respuestas a las anteriores preguntas pueden inclinarse más en un sentido afirmativo o negativo, aunque su mayor y más claro empeño se evidencie en ofrecernos una respuesta negativa.

Por un lado, encontramos a un Jakobs que separa tajantemente los espacios de la política criminal y la dogmática jurídico penal. En efecto, expresiones como las que se citarán a continuación nos dan esa impresión:

- "Quien sólo sabe que una sociedad esta organizada de modo funcional, no sabe nada acerca de su configuración concreta». ${ }^{12}$

10 Roxin, Claus. La evolución de la política criminal, el derecho penal y el proceso penal. Valencia: Tirant lo Blanch, 2000, p. 69.

11 DonniNı, Massimo. \«Un derecho penal fundado en la Carta Constitucional: razones y límites. La experiencia italiana\», en: Revista Penal, julio, nº 8, 2001.

12 JakовS, Günter. Sociedad, norma y persona en una teoría de un derecho penal funcional, ob. cit., p. 36. 
- «La decisión sobre el alcance de los procesos de criminalización sería una tarea puramente política, no jurídico penal». ${ }^{13}$

- «Si la sociedad realmente se desliza hacia una limitación de los derechos de la libertad, eso no es sólo en el derecho penal [...]. Si se trata de un exceso de criminalización innecesario o de la necesaria defensa de un contenido mínimo, sólo puede determinarse en un plano político, pero no al nivel de la ciencia del derecho penal». ${ }^{14}$

Jakobs es plenamente consciente de las consecuencias de esta tesis cuando sostiene que «[...] probablemente esta descripción neutra, esta exclusión de la utopía, es lo más chocante en la práctica de toda la teoría funcional». ${ }^{15}$

Mussig, uno de sus discípulos más cercanos, refuerza estas ideas cuando afirma que las cuestiones de legitimidad material de las normas penales desbordarían el cauce formal (y positivista) de la prevención general positiva y remitirían al análisis de los criterios de identidad de una determinada sociedad. ${ }^{16}$

Pues bien, estas y otras afirmaciones nos conducirían a coincidir con aquellos que cuestionan el alto grado de formalización de la construcción jakobsiana, formalización que, a decir de Roxin, desvincularían el fundamento de la dogmática de los fines de la pena propios de un Estado social y democrático de derecho. ${ }^{17} \mathrm{La}$ única referencia funcional valorativa se encontraría en la función estabilizadora de la norma penal, lo que demuestra el denunciado círculo vicioso en la metodología interpretativa jakobsiana, ${ }^{18}$ el mismo que conduciría a restringir las facultades del operador judicial frente a los excesos del legislador penal. ${ }^{19}$ Esta restricción, querida por el funcionalismo sistémico, no es nuestra

13 Jakobs, Günter, ZStW 107 (1995), p. 855, tomado de Peñaranda Ramos, Enrique; Suarez Gonzales, Carlos; y Cancio Melia, Manuel. "Consideraciones sobre la teoría de la imputación de Günter Jakobs», ob. cit., p. 28.

14 JаковS, Günter. Sociedad, norma y persona en una teoría de un derecho penal funcional, Madrid: Civitas, 1996, p. 40.

15 Ibíd., p. 67.

16 Peñaranda Ramos, Enrique; Suarez Gonzales, Carlos; y Cancio Melia, Manuel. «Consideraciones sobre la teoría de la imputación de Günter Jakobs», ob. cit., p. 28.

17 Roxin, Claus. La evolución de la política criminal, el derecho penal y el proceso penal, ob. cit., p. 66.

18 Schunemann, Bernd. «Sobre la crítica a la teoría de la prevención general negativa». En Política criminal y nuevo derecho penal. Libro homenaje a Claus Roxin. Barcelona: Bosch, 1997, p. 92.

19 De esta apreciación es Portilla Contreras, Guillermo. «Los excesos del formalismo jurídico neofuncionalista en el normativismo del derecho penal». En Mutaciones del Leviatán. Legitimación de los nuevos modelos penales. Madrid: Ediciones Akal, 2005, p. 59. El autor refiere textualmente con respecto a la tesis de Jakobs que «[...] basándose en un principio de prevención general positiva orientado al mantenimiento del sistema social su pensamiento se erige en un perfecto círculo en el que no se cuestiona las decisiones del subsistema político creador del Derecho». 
opción para un Poder Judicial como el peruano el cual ha padecido históricamente de déficit de poder. ${ }^{20}$

\section{El peligroso referente externo en Jakobs}

Jakobs ha pretendido matizar esta alta formalización de su sistema, señalando que una cosa es «[...] la autosuficiencia del sistema jurídico [...] y otra cuestión distinta es qué problema del sistema social se resuelve mediante la génesis diferenciada de normas específicamente jurídicas y en definitiva, mediante la génesis diferenciada de un derecho jurídico en especial». Para esta última, esto es, para el trabajo dogmático, la perspectiva externa al sistema jurídico debe ser tomada en cuenta como presupuesto para la perspectiva interna. Según Jakobs la sociedad es esa perspectiva externa a tener en cuenta durante el trabajo dogmático. Sin embargo, dicha referencia a lo social no es, según entiendo, una referencia al modelo social que orienta las normas iusfundamentales, esto es, el consenso social básico normativizado en la Constitución, sino esencialmente una referencia a las demandas sociales coyunturales de lo justo. ${ }^{21}$ Es por ello que Schunemann ha resaltado los riesgos de una concepción preventiva de la pena como la de Jakobs en el sentido de la influenciabilidad del sentimiento de justicia de la colectividad en las medidas adoptadas en función de dicho sentimiento. En palabras del propio Schunemann:

Si la sanción de un delincuente no es en sí misma conforme con el principio de justicia, aunque la colectividad, en virtud de sus arcaicas representaciones, mantenga que lo es, no podrá seguir siendo justo recurrir a las mismas como concepto al servicio de la prevención general positiva. Si así fuera la justicia penal [...] fundamentaría la pena — que en calidad pasaría a ser injusta— en unas concepciones sociales superadas. Con ello se abusaría del delincuente como medio para satisfacer anticuados prejuicios, lo cual conduciría a una hipocresía; e incluso con esa justificación se impediría cualquier efectividad de la pena. ${ }^{22}$

Pero por otro lado, Jakobs ha efectuado algunas otras afirmaciones que oscurecen la claridad de sus postulados anteriores. Son generalmente afirmaciones sostenidas en el contexto de sus respuestas a las críticas que atribuyen a su

20 Roxin, Claus. La evolución de la política criminal, el derecho penal y el proceso penal, ob. cit., p. 52.

21 Claramente sobre este aspecto SiLva SÁnCHEZ, Jesús María. Aproximación al derecho penal contemporáneo, ob. cit., pp. 235 y 236, quien además, replica convincentemente a aqueIlos que ven una mayor idoneidad de la tesis preventivo estabilizadora sobre la tesis preventivo general negativa con relación a sumir en su seno los principios limitadores de la intervención penal.

22 SCHNEMANN, Bernd. «Sobre la crítica a la teoría de la prevención general positiva», ob. cit., p. 91. 
sistemática un contenido conservador, tecnocrático y, por ello mismo, funcional a cualquier movimiento de expansión del sistema penal y promotora de la reproducción ideológica material de las relaciones sociales existentes. ${ }^{23}$ Jakobs, si bien reconoce que su perspectiva funcional (neutral) es indistintamente aplicable a una sociedad esclavista como totalitaria, advierte también que sus conclusiones obtenidas de problemas concretos del sistema social «[...] se deben en parte a las particulares condiciones del derecho vigente en Alemania, pero tomado no sólo en su calidad de derecho positivo, sino también de ordenamiento legítimo, produciéndose de este modo [...] una cierta síntesis entre justificación funcional y una legitimación racional valorativa». ${ }^{24}$ Esta dogmática real, como denomina Roxin a las conclusiones dogmáticas obtenidas por Jakobs, es en parte semejante a las conclusiones elaboradas desde el funcionalismo valorativo. Esta no infrecuente similitud en los resultados dogmáticos se debe, según el propio Roxin, a que Jakobs «[...] aplica al derecho penal vigente el procedimiento metodológico y el instrumental conceptual de la teoría social sistémica pero cuyos contenidos son deducibles de la ley y de la Constitución». ${ }^{25}$ La pregunta que surge necesariamente en este punto es por qué Jakobs no ha derivado toda su construcción sistemática de esa autodescripción de la sociedad plasmada en la Constitución alemana y en los documentos internacionales de derechos humanos. Por lo menos dos serían las explicaciones a esta interrogante.

Una primera nos la ofrece Roxin cuando refiere que en la construcción de Jakobs todo se contempla aún desde la perspectiva del sistema dominante y no desde el sistema que, siendo aún minoría, postula eventuales reformas político criminales. La segunda explicación, creo yo, radica en el escaso rendimiento que la ciencia penal alemana ha reconocido a la posibilidad de integración de los derechos y principios constitucionales y del derecho internacional de los derechos humanos en la sistemática del derecho penal. La influencia de la Constitución en las normas penales se limita prácticamente al marco general que ofrece el modelo de Estado reconocido por la Constitución alemana y no a la interacción que los diversos derechos fundamentales y

23 Baratta, Alessandro. I Integración - prevención: una nueva fundamentación de la pena dentro de la teoría sistémica \», en Cuadernos de Política Criminal, nº 24, 1984.

24 Peñaranda Ramos, Enrique; Suárez Gonzáles, Carlos; y Cancio Melia, Manuel. «Consideraciones sobre la teoría de la imputación de Günter Jakobs», ob. cit., p. 29.

25 Roxin, Claus. La evolución de la política criminal, el derecho penal y el proceso penal, ob. cit., p. 54. Este autor observa, además, que el proceso de imputación preventivo general de Jakobs comprende premisas como las de la libertad y la dignidad personal. Ver también JAKOBS, Günter. Sociedad, norma y persona en un derecho penal funcional, ob. cit., pp. 29 y 30, cuando sostiene que «[...] la cuestión del sujeto libre estará presente exactamente en aquella medida en la que sea trasmitido por medio de la comunicación, es decir, en la medida en la que sea determinante de la autodescripción de la sociedad [...] [así] puede suceder que se convierta en el objeto central de la comunicación y entonces será el elemento dominante, por encima de todo lo demás». 
otros intereses reconocidos en la Constitución pueden y deben ofrecer al funcionamiento y contenido material del derecho penal.

\section{La ausencia de verificabilidad empírica de la justificación del derecho penal en la tesis jakobsiana}

Pasando a otro aspecto de la tesis preventiva de Jakobs, debo manifestar que, según mi apreciación, la naturaleza utilitaria de esta no aparece lo suficientemente determinada. Ello evidentemente ha dado pie a considerar la tesis preventivo general positiva como una forma renovada del retribucionismo hegeliano. ${ }^{26}$ Atribución nada descabellada cuando el propio Jakobs ha admitido que la concepción de ese filósofo no difiere en mucho de su propia concepción. ${ }^{27}$

En efecto, la cuestión a dilucidar es si la función preventivo general positiva como fin del derecho penal, remite a un efecto real sobre los ciudadanos o es solo un argumento de legitimación abstracta al margen de sus consecuencias en la realidad. En otras palabras, ¿la finalidad preventivo integradora del derecho penal pretende efectivamente prevenir conductas lesivas a la convivencia democrática? Tal como indicamos anteriormente, una de las exigencias del Estado social y democrático de derecho es su efectiva contención del delito.

Pues bien, en este aspecto, Jakobs, no es tampoco uniforme, habiendo cambiado sustancialmente los elementos de su tesis por lo menos en dos ocasiones. En un primer momento Jakobs ${ }^{28}$ destacó como elemento central de su tesis los efectos psicológicos que la pena eventualmente produce sobre los individuos o la sociedad, efectos que no son otra cosa que el aprendizaje en la intolerabilidad del comportamiento delictivo. En palabras del propio Jakobs: «Esto es prevención general no en sentido intimidatorio sino como ejercicio de la fidelidad hacia el Derecho». ${ }^{29}$

En esta fase de evolución de la tesis jakobsiana la pena aún mantenía su vocación utilitaria. De hecho, si tales efectos empíricos se pretendían producir con la conminación y la aplicación de la pena, resulta lógico pensar en una necesaria y querida reducción de las conductas infractoras de la norma penal. Sin embargo, a pesar del utilitarismo de la primera versión de la tesis de Jakobs, esta fue objeto de severas críticas que influyeron en su replanteamiento.

26 Silva SÁnchez, Jesús María. Aproximaciones al derecho penal contemporáneo, ob. cit., p. 205

27 Citado por Peñaranda Ramos, Enrique; Suárez Gonzáles, Carlos; y Cancio Melia, Manuel. "Consideraciones sobre la teoría de la imputación de Günter Jakobs», ob. cit., p. 24.

28 JAKOBS, Günter. «Culpabilidad y prevención». En Estudios de derecho penal. Madrid: Civitas, 1997, p. 79.

29 Ibíd., p. 79. 
Efectivamente, de acuerdo con esta primera versión, resulta inevitable la proyección del derecho penal sobre la esfera interna del ciudadano a fin de provocar en él una actitud de fidelidad al Derecho. ${ }^{30}$ Esto último, como es evidente en una sociedad democrática, implica una intolerable legitimación del derecho penal por medio de finalidades ético-sociales. ${ }^{31}$

Lo intolerable de esta finalidad en un Estado democrático de derecho resulta en virtud de por lo menos dos consideraciones fundamentales: en primer lugar, porque una intervención penal en la dirección de la conciencia interna del ciudadano vulnera las exigencias propias de una sociedad democrática, la misma que, como principio básico, prescribe la coexistencia plural y pacífica de convicciones, opiniones e ideas entre los miembros que la componen, incluso cuando alguna de esas convicciones u opiniones discrepen con el marco de principios que la Constitución política reconoce.

En segundo lugar, porque la intromisión en la esfera interna del individuo atenta contra el principio de respeto a la dignidad humana, sobre todo en una de sus manifestaciones fundamentales como es el principio de autonomía de la persona. Es decir, la dignidad humana exige del Estado y de terceros, el reconocimiento de un espacio privado propio de auto organización humana a fin de permitir el libre desarrollo de su personalidad (artículo 10. de la CE).

Pero, como hemos mencionado, el profesor Jakobs hizo algunos cambios en su teoría, los cuales fueron plasmados en su tratado de derecho penal. ${ }^{32}$ En términos resumidos estos cambios supusieron la accesoriedad de los efectos psicológicos como componentes de su tesis preventiva y la centralidad de la idea de la pena como garantía para el mantenimiento de la confianza en la

30 Jaковs, Günter. Derecho penal. Parte general, ob. cit., pp. 14 y 18, quien luego de señalar que la «[...] misión de la pena es el mantenimiento de la norma como modelo de orientación para los contactos sociales» y que «[...] los destinatarios de la norma no son primariamente algunas personas en cuanto autores potenciales», indica «En esta medida la pena tiene lugar para ejercitar en la confianza en la norma [...]».

31 BARATTA, Alessandro: «Integración - prevención: una nueva fundamentación de la pena dentro de la teoría sistémica», ob. cit., p. 538 y ss, desde el plano de la culpabilidad, ha mostrado cómo a pesar de la apariencia tecnocrática de las posturas de Jakobs (quien señala que el fundamento funcionalista de la petición social de la pena como criterio normativo para la determinación de la responsabilidad penal es independiente del marco conceptual de la teoría del inconsciente colectivo), estas también continúan utilizando un criterio social y funcional de adscripción de responsabilidad penal para «[...] la estabilización del ordenamiento y de la confianza en él». Siıva SÁNCHEz, Jesús María. Aproximaciones al derecho penal contemporáneo, ob. cit., p. 236, también ha mostrado la inevitable contradicción que se presenta entre las garantías personales y los intereses sociales por lo que la prevención general positiva utiliza inevitablemente criterios psicológico sociales basados, en determinados casos, en profundos e inconscientes sentimientos irracionales de venganza social. También en esa línea, como hemos visto, SchunemanN, Bernd. "Sobre la crítica a la teoría de la prevención general positiva», ob. cit., pp. 91 y 92.

32 Jakoвs, Günter. Derecho penal. Parte general, ob. cit., pp. 9-19. 
vigencia de la norma. ${ }^{33}$ De acuerdo con ello la pena constituye una respuesta (contradicción) al quebrantamiento de la norma por lo que esta, a pesar de su quebrantamiento, sigue siendo la regla de comportamiento en que se puede seguir confiando. ${ }^{34}$ Bajo esta perspectiva, entonces, la tesis preventivo general positiva pierde toda referencia empírica o por lo menos ella deja ser su necesaria consecuencia. Así lo ha afirmado el propio Jakobs:

La pena no es sólo un medio de mantenimiento de la identidad social, sino que representa ese mantenimiento. El restablecimiento de la identidad social no es la consecuencia del castigo, sino su significado. Ciertamente puede que se vinculen a la pena determinadas consecuencias de psicología social o individual de muy variadas características. Pero la pena ya significa algo con independencia de las consecuencias: significa una autocomprobación. Por ello las investigaciones empíricas relativas a la prevención general positiva están siempre un poco fuera de lugar. ${ }^{35}$

Desde nuestro punto de vista, un postulado legitimante del derecho penal debe ser susceptible de algún grado de verificabilidad empírica. Esta posibilidad, aunque difícil, es una de las garantías para el control de legitimidad político criminal de las normas penales. Solo por medio del análisis de las consecuencias de la pena es posible descubrir y denunciar las normas penales meramente simbólicas, muchas veces encubridoras de la ausencia de políticas públicas las cuales bien llevadas podrían resultar más eficaces que la propia pena. ${ }^{36}$

\section{La pérdida de especificidad del derecho penal como mecanismo de control social formalizado}

En tercer lugar, $\mathrm{y}$ dentro de mis comentarios a la tesis preventivo general positiva de Jakobs, considero que no ha sido respondida satisfactoriamente la crítica que Mir Puig dirige a dicha tesis en el sentido de que desde aquella perspectiva no es posible explicar «[...] por qué la estabilización de las expectativas ha de tener

33 Peñaranda Ramos, Enrique; Suárez Gonzáles, Carlos; y Cancio Melia, Manuel. «Consideraciones sobre la teoría de la imputación de Günter Jakobs», ob. cit., p. 20.

34 Ibíd., p. 21

35 Jakoвs, Günter. Sociedad, norma y persona en un derecho penal funcional, ob. cit., p. 18. Es por ello que un discípulo del referido profesor alemán, H. Lesch, ha preferido rechazar la denominación de prevención general positiva y optar por la de «teoría funcional de la retribución».

36 Al respecto BARATTA, Alessandro. «Integración - prevención: una nueva fundamentación de la pena dentro de la teoría sistémica», ob. cit., p. 546, sostiene que esta tesis desconoce el hecho de que el sistema produce altos costes sociales y gravísimos efectos sobre la integración social y la confianza en las instituciones. Ofrece en consecuencia una visión parcial de los reales efectos que produce la pena: solo presenta los efectos positivos ocultando los negativos. 
lugar (necesariamente) por medio de la imposición de una pena y no por otros medios menos lesivos y funcionalmente equivalentes». ${ }^{37}$ En efecto, la tesis de Jakobs no nos ofrece criterio alguno que nos permita optar adecuadamente entre un control social informal o formal extrapenal y un control formal punitivo. Todos estos tipos de control tendrían entre sus efectos — en mayor o menor medida - la estabilización de las normas de convivencia social. Esta indiscriminada función estabilizadora de las múltiples formas de control social impide atribuirle a la prevención general positiva el carácter de fundamento o finalidad legitimadora por excelencia del derecho penal, en tanto mecanismo especial del sistema de control social formalizado.

Peñaranda Ramos, Suárez Gonzáles y Cancio Melia ${ }^{38}$ han pretendido matizar estas críticas, señalado que Jakobs ha tenido y tiene en consideración estrategias de solución de conflictos alternativas a la pena. Así por ejemplo, se refiere, que Jakobs en su trabajo Culpabilidad y prevención sostiene que "[...] la necesidad de pena decae cuando resulta posible un procesamiento distinto de la defraudación de la expectativa» o cuando en su tratado de derecho penal hace mención a "[...] otras posibilidades de solución del conflicto». ${ }^{39}$ Sin embargo, como ellos mismos reconocen, solo se trata de establecer que Jakobs las tiene en cuenta, más no explican cómo Jakobs deduce de su tesis de la prevención general positiva la idoneidad de recurrir a otras medidas alternativas a la pena. Incluso el propio Müssig ${ }^{40}$ ha reconocido esta limitación de la prevención general positiva y ha postulado la necesidad de recurrir a otros criterios generalmente establecidos en la Constitución y en especial a los derechos fundamentales en él reconocidos. ${ }^{41}$ Se recurre nuevamente a determinados criterios externos a pesar de que Jakobs y sus discípulos han criticado esta postura como un déficit de la tesis preventivo general negativa. ${ }^{42}$ Pues bien, queda evidenciada la necesidad de recurrir a los elementos garantistas reconocidos en la Constitución Política al momento de modular la oportunidad e idoneidad

37 Expresivamente MIR PUIG, Santiago, en «Función fundamentadora y función limitadora de la prevención general positiva», ob. cit., se pregunta por qué no habría de bastar con una declaración inequívoca al respecto y por qué es preciso imponer un mal como pena si esta no busca la intimidación sino evitar posibles dudas sobe la vigencia de la norma infringida.

38 Peñaranda Ramos, Enrique; Suárez Gonzáles, Carlos; y Cancio Melia, Manuel. Un nuevo sistema del derecho penal: consideraciones sobre la teoría de la imputación de Gunter Jakobs. Bogotá: Universidad Externado de Colombia, 1999.

39 JakовS, Günter. Estudios de Derecho Penal, Civitas, Madrid, 1997.

40 Tomado de Peñaranda Ramos, Enrique; Suárez Gonzáles, Carlos; y Cancio Melia, Manuel. Un nuevo sistema del derecho penal: consideraciones sobre la teoría de la imputación de Gunter Jakobs, ob. cit., pp. 31 y 32.

41 Jakobs, Günter. Derecho penal. Parte general, ob. cit., p. 13. También hacen mención a la necesidad de Jakobs de acudir a criterios externos como el de subsidiariedad PEÑARANDA Ramos, Enrique y otros, ob. cit., p. 32.

42 Ibíd., p. 76. 
de la conminación penal abstracta o su aplicación concreta. Este es otro de los aspectos en los que el reconocido autor alemán se acerca a los postulados de aquellos que pretendemos operar con un derecho penal de corte funcional valorativo.

\section{Conclusión}

Concluyendo entonces, consideramos que la tesis preventivo general positiva resulta insuficiente como teoría legitimadora preferente o exclusiva de la intervención punitiva del Estado. Ello, en primer lugar, por que prescinde de sustanciales principios constitucionales de garantía de la intervención punitiva en el trabajo dogmático funcional, rebajando de esta manera la potestad que le corresponde a la jurisdicción en el marco de un Estado democrático constitucional asentado sobre el principio del control de poderes. La referencia social a la cual nos remite la tesis en cuestión no es el modelo social normativizado de la constitución y la vigencia de los derechos fundamentales, sino el coyuntural sentimiento social y político imperante de lo justo, muchas veces cargados de prejuicios cotidianos y sentimientos de venganza social.

En segundo lugar, la insuficiencia de la tesis preventivo general positiva viene motivada en la renuncia, por lo menos en la versión acabada de la tesis de Jakobs, de criterios de verificación empírica de los efectos del derecho penal, lo que la convierte en una justificación ajena a las exigencias utilitarias de un Estado social y democrático de derecho. Y finalmente, por que en sí misma la referida tesis carece de rendimiento para discriminar el tipo de medidas (punitiva u otras alternativas menos violentas) adecuadas frente a una determinada conducta disfuncional. Su necesaria recurrencia a criterios externos nos muestra la conveniencia explícita de recurrir a criterios normativo constitucionales que legitimen el derecho penal y no a tesis con pretensiones de autosuficiencia.

Coincidimos por ello con Schunemann cuando concluye que "[...] la prevención general positiva es un bienvenido efecto concomitante, pero carente de significado teorético propio». ${ }^{43}$ Efectivamente, no puede negarse que el derecho penal cumple determinados efectos de «[...] integración y estabilización social que pasa por la reafirmación simbólica de la norma». ${ }^{44}$ Pero tales efectos, en el plano empírico, no pueden constituirse en el fin legitimador del derecho penal, aunque siempre será necesario tenerlos en cuenta como parte de su funcionamiento.

43 SChunemann, Bernd. «Sobre la crítica a la teoría de la prevención general positiva», ob., cit., p. 98.

44 Silva Sánchez, Jesús María. Aproximaciones al derecho penal contemporáneo, ob. cit., p. 233. 